\title{
Performance Improvement of Mobile Ad-hoc Networks through Energy Conservation Scheme
}

\author{
Poonam Shrivastava \\ Technocrates Institute of \\ Technology, Anand Nagar \\ Bhopal, India
}

\author{
Amit kumar Sinhal \\ Technocrates Institute of \\ Technology, Anand Nagar \\ Bhopal, India
}

\author{
Abhishek Gupta \\ TRUBA Institute of Engineering \& \\ Information Technology \\ Bhopal, India
}

\begin{abstract}
MANET is a collection of mobile node in a wireless network having communication between them without any centralized control or established infrastructure.

Energy consumption in the wireless interface of the mobile node can be eliminated or reduced. Here, we have discussed various energy awareness schemes responsible for energy optimization.

The main goal of the proposed methodology, is to create a new scheme based on energy conservations, that helps each and every node to save its energy to certain extent and so that it can actively participate in the transmission process for the long term, thus to reduce the idle energy consumption within the network. The algorithms of all categories are compatible with such scheme. These functionality strategies are architecture of the routing protocol.
\end{abstract}

Here, a new model has been developed that is elaborated form of AODV protocol based on energy conservation scheme. i.e EAODV.

Thus in this paper, the focus is on methods used in energy based algorithms to reduce the power consumed in communications between ad hoc network nodes.

\section{Keywords}

MANET, AODV, Energy, TCP, E-AODV;

\section{INTRODUCTION}

A wireless Adhoc network which can be established by wireless computer (nodes) is a temporary network. In such types of networks no infrastructure is required such as base stations and access points. Since no base station are supported in such an environment and mobile host communicate in a multi-hop fashion thus temporary network connectivity is required such as in battlefields, disaster areas, and meetings, because of their capability of handling node failures and fast topology changes[1].

This paper addresses the issue of energy-conserving routing protocols in ad hoc networks of mobile hosts. Adhoc networks are multi-hop, wireless networks where all mobile hosts or nodes cooperatively maintain network connectivity without communication infrastructures for routing. Many different routing protocols have been proposed in the literature and submitted to the Internet Engineering Task Force (IETF) Mobile Ad Hoc Network (MANET) Group. A major issue in these algorithms is to find a shortest path consisting of minimum number of intermediate forwarding nodes between a source and a destination. However, it is possible that some particular mobile nodes are unfairly burdened to support many packet-relaying functions. This hot spot node may consume more battery energy and stops running earlier than other nodes disrupting the overall ad hoc network. This is particularly true for some optimized routing protocols that prefer specific mobile nodes in the selection of routing paths [2].

\section{AODV}

The ad hoc mobile network uses a routing protocol known as Ad hoc On Demand Distance Vector (AODV) routing protocol. AODV can be used for both unicast and multicast routing. It is known as On-demand routing algorithm because routes are established between nodes only as desired by source nodes and maintain them as long as they are needed by the sources. In AODV the sequence numbers of the nodes are used to ensure the freshness of routes.

Under highly dynamic link conditions, reactive protocols are expected to generate fewer overhead messages and provide a more reliable routing than proactive routing protocols.

Route discovery and route maintenance for AODV are described below.

\subsection{Route Discovery}

Whenever a traffic source needs a route to a destination the route discovery process is initiated. Route discovery typically involves a network-wide flood of route request (RREQ) packets targeting the destination and waiting for a route reply (RREP). An intermediate node receiving a RREQ packet first sets up a reverse path to the source using the previous hop of the RREQ as the next hop on the reverse path. If a valid route to the destination is available, then the intermediate node generates a RREP, else the RREQ is re-broadcast. Duplicate copies of the RREQ packet received at any node are discarded. When the destination receives a RREQ, it also generates a RREP. The RREP is routed back to the source via the reverse path. As the RREP proceeds towards the source, a forward path to the destination is established.

\subsection{Route Maintenance}

Route maintenance is done using route error (RERR) packets. When a link failure is detected, a RERR is sent back via separately maintained predecessor links to all sources using that failed link. Routes are erased by the RERR along its way. When a traffic source receives a RERR, it initiates a new route discovery if the route is still needed. Unused routes in the routing table are expired using a timer-based technique.

\section{ENERGY CONSUMPTION IN AODV NETWORK}

Energy consumption in AODV network composed of different sources. Since the rate of battery performance improvement is rather slow currently, and in the absence of breakthroughs in this field, other measures have to be taken to achieve the goal of getting more performance out of the currently available battery resources. So, in our study, we focus our efforts on methods to reduce the power consumed in communications between ad hoc network nodes. 
Various studies have been done to determine the exact sources of energy consumption in the wireless interfaces.

Following are the types of energy consumption that have been identified:

$>$ Energy consumed while sending a packet.

$>$ Energy consumed while receiving a packet

$>$ Energy consumed while in idle mode.

$>$ Energy consumed while in sleep mode.

It is noted that the amount of energy that is consumed in the receive operation is comparatively less than compare to the amount that is consumed in sending a packet.

Thus in these studies it has been shown [7] that energy consumed in the retransmit operations is responsible for a considerable amount of energy consumption.

\section{ENERGY CONSUMPTION OVERHEAD}

Energy consumption measurements studies have been conducted to determine the power consumption patterns in the different active modes. In these experiment, the instantaneous power consumption per communication mode, e.g. send, receive, idle and sleep modes, has been measured.

Various sources of energy consumption or energy wastage in wireless network can be attributed to the following.

$>$ Idle condition

$>$ Collisions

$>$ Protocol overhead

Energy consumed during node communications will be focused on some studies, e.g. [8] discuss metrics for energy efficiency in ad hoc networks.

\section{PROPOSED METHDOLOGY}

Since, there are various techniques to reduce the energy consumption during the transmission of data packet in the transmission process.

In proposed methodology, a technique is proposed to reduces energy consumption in communication in mobile nodes over the previously used techniques-

Here in proposed methodology a new protocol E-AODV over the previously used AODV protocol have been developed, that is based on energy conservation or energy consumption scheme thus to save the energy of each node in the transmission process so that each node can survive in the network for the longer time and can participate active in wireless Adhoc network.

This comparison can be done on basis of distinct metrics.

To compare the energy-efficiency of a routing protocol we can use several distinct metrics. The selection of appropriate metrics depends on the overall goal of the ad-hoc network deployment. Various types of metrics that are used to manage energy consumption are:

$>$ Minimize energy consumed / packet,

$>$ Maximize time to network partition,

$>$ Minimize variance in node energy levels,

$>$ Minimize cost/packet, and

$>$ Minimize maximum node cost.

The "Minimize energy consumed/packet" metric basically addresses the average energy consumed per packet, over the number of hops that are traversed by this packet [6].

Hence we can calculate the energy consumed for packet $\mathrm{x}$, which are transmitted from source to destination over $\mathrm{m}$ hops, is:

$$
€=\text { ẹị, } i+1
$$

Where $€$ is the energy consumed for packet $x$, ệ is the energy consumed in transmitting one packet between two nodes.
For the "Minimize cost/packet" metric there is need to define a cost function, that is the total cost (or energy) of sending a packet through the same path.

The analysis and result of analysis can be easily understood. The result shows the improvement of new proposed algorithm (EAODV) over the previously used algorithm AODV to reduce the energy consumption take place during the transmission of data packet in wireless adhoc network.

In the given methodology, the decrement in the amount of energy consumption is visualized on the bases of variance which is calculated on the basis of amount of energy lost by each node during transmission and thus taking the mean of all the lost energy. The calculation for Variance is shown as:

$$
¥+=(£-\S) *(£-\S) / \varnothing
$$

Where, $¥$ = variance

$£=$ Energy Remaining.

$\S=$ mean of total energy consumption per node.

$\phi=$ count is the total number of node taking active participation in transmission.

Where the energy remaining can be calculated through

$$
£=\xi / \varnothing
$$

$\xi=$ Energy consumption per node, $\phi=$ Count number of active nodes.

Thus on the basis on variance we can calculate the number of active node, and the node in died state.

Thus in given methodology as the variance calculated is reduced as compared to the previous one. Thus reducing the number of nodes in died state.

As the amount of active time of per node increase, hence more amount of data can be send with less amount of energy consumption in wireless adhoc network.

Here in energy aware AODV i.e. E-AODV the unbalanced energy consumption of any particular node can be reduced considering the residual energy of any node along with the hop count. Thus, it balanced the node energy consumption and extends the life time of the network.

In E-AODV protocol, the unbalanced energy consumption of the node is balanced by considering the residual energy of that node along with the hop count of that particular node.

Here, actually the energy of the entire particular node is saved so that it can participate in the network transmission process for the longer time and so as to extend the life time of overall network without performance degradation in a much better as compare to the AODV protocol.

The optimal route is determined by using the value of parameter $\alpha$, as we have describe in the equation given below [5],

$$
\alpha=\frac{\text { Min }- \text { R.E }}{\text { \#Hops }}
$$

Where,

Min - RE is the minimum residual energy on the route.

\#Hops is the hop count of the route between source and destination belonging to that node. 
The value of $\alpha$ is calculated by the destination node for any of the received routes. So that it can chooses a route with the largest value of $\alpha$.

Thus in the given energy awareness, EAODV protocol minimum residual energy of any particular nodes is collected which is relatively large and have the least hop-count, and then on the basis of the information provided we determines a proper route among them, which consumes the minimum network energy compared to any other routes [5].

\subsection{Proposed Algorithm for the Analysis of E- AODV Routing Protocol}

The operations of the E-AODV Routing protocol can be understand with help of following algorithmic steps:

Step 1: If any of the node, wants to send some data packets

It generates the route request (RREQ) packet and broadcast it among its neighbor nodes.

Step 2: Each of its neighboring nodes, which receives (RREQ) sends Route Reply (RREP) packet back to its source nodes,

These reply packets contain two fields, Lx \& Ly, containing hop counts (\#Hop) and minimum residual energy of that node (Min. RE)

Step 3: In E-AODV, each node waits for a time (T_wait) till it receives all the RREP messages from its neighboring nodes.

\section{SIMULATION RESULTS}

The simulation is done in NS-2.34 environment on Linux operating system (Ubuntu10.04). In our work the utilities given in the ns-2.34 such as AWK, XGRAPH, GNUPLOT, TCL and we have also used $\mathrm{C}++$. Two types of files such as trace file and nam file are generated during the simulation process. Trace file is used for getting information about the network.

\subsection{Simulation Parameter Used}

The simulation consists of a network of 50 nodes confined in a $1500^{\prime} 300 \mathrm{~m}^{2}$ area. Random connections were established using CBR traffic (at 3 packets/second with a packet size of 512 bytes). The initial battery capacity of each node is 10 units. This initial energy is progressively reduced by data transmission/reception. When it reaches zero units, the corresponding node cannot take part any more in the communication, and is regarded as died.

Table 1.1: Simulation parameters for the case study

\begin{tabular}{|c|c|}
\hline Simulator Used & NS-2.34 \\
\hline Number of nodes & 50 \\
\hline Dimension of simulated area & $1500 \mathrm{~m} \times 300 \mathrm{~m}$ \\
\hline Routing Protocol & AODV \\
\hline Simulation time & $600 \mathrm{Sec}$ \\
\hline Traffic type & CBR $(3 \mathrm{pkts} / \mathrm{s})$ \\
\hline Packet size & 512 bytes \\
\hline No. of traffic connections & TCP / UDP \\
\hline Node movement at maximum & Random \\
\hline Speed (m/s) & $250 \mathrm{~m}$ \\
\hline Transmission range & 10 joule \\
\hline Threshold value & 1.5 joule \\
\hline Transmit power & 1.0 joule \\
\hline Receiving power & .17 joule \\
\hline Idle power & .047 joule \\
\hline Sleeping power &
\end{tabular}

Table 1.2: Energy model parameters for the case study

\begin{tabular}{|c|c|}
\hline Rx Power Consumption & $1.0 \mathrm{~W}$ \\
\hline Tx Power Consumption & $1.4 \mathrm{~W}$ \\
\hline Idle Power Consumption & $0.83 \mathrm{~W}$ \\
\hline
\end{tabular}

\subsection{Energy level of nodes}

The figure given below, analyzes our result through energy discharge graph, basically node energy discharge through transmission power, receiving power, ideal power and sleep power etc. if node energy less the 10 joule that means node are survival condition and route will updated means route change and if energy higher than the threshold 10 joule so new route establish. Graph shows life time and energy discharge each node our life time is 100 second because energy utilize till end of simulation time and node survival condition after 90th second.

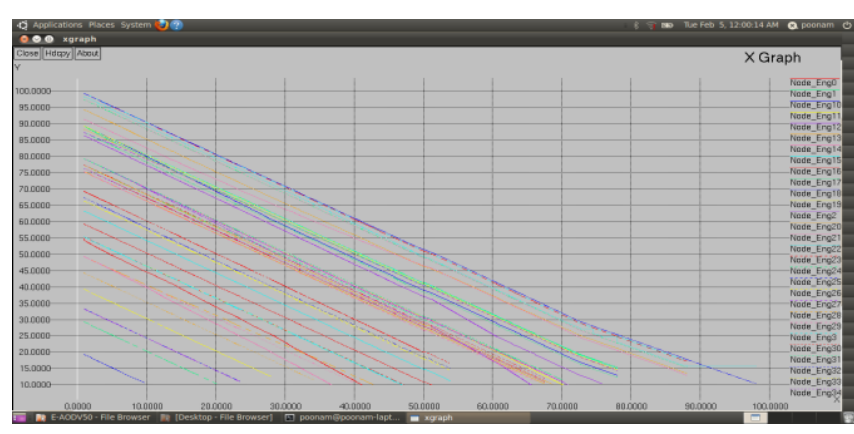

Figure 1. Scenarios represent the different energy state level in time $100 \mathrm{~ms}$

\subsection{TCP Analysis}

Here we present TCP flow graph at the time of energy aware technique applies with AODV routing protocol, here we create ten TCP connections and analyze our result. Graph shows maximum TCP transfer through connection number seven and lower transmission through tenth connection. $\mathrm{X}$-axis represent Time unit per/s and Y-axis represent No. of packets transfer by the connection.

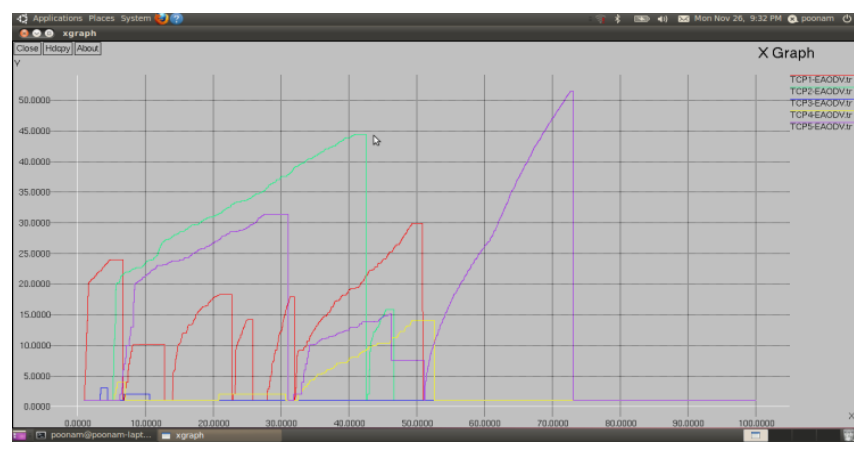

Figure 2. Figure representing 5 TCP connections

\section{Conclusion \& Future Work}

In this paper, the sources of energy consumption and communications in ad hoc network were shown to exist in four main modes of operation.

Here we have discussed the energy consumption overhead such as idle condition, collisions and protocol control messages and also focus our attention on energy-efficiency strategies done through various metrics.

Our ongoing work is aimed to achieve the best possible fulfillment of performance expectations, though the energy 
management done at different levels of an ad hoc sensor network. The implementation in our routing protocol can also be extended in future by applying the same algorithm on various other routing protocols such as DSR, DSDV, OLSR, TORA protocol inspite in AODV protocol and characteristic all the routing protocols can be compared.

\section{REFERENCES}

[1] S.-M. Senouci and G. Pujolle, "Energy Efficient Routing in Wireless Ad Hoc Networks", Laboratoire LIP6 - Université de Paris VI, France.

[2] K. Woo, C. Yu, D. Lee, H. Y. Youn, and Ben Lee, "NonBlocking, Localized Routing Algorithm for Balanced Energy Consumption in Mobile Ad Hoc Networks", AB._C\#D_E _ CGF $>$ H , Cincinnati, Ohio, August 15-18, pp. $117-124,2001$.

[3] J. Broch, D. A. Maltz, D. B. Johnson, Y.-C. Hu and J.Jetcheva, "A Performance Comparison of Multi-Hop Wireless Ad Hoc Network Routing Protocols, "International Conference on Mobile Computing and Networking (MobiCom'98), Oct. 1998.
[4] S. Corson and J. Macker, "Mobile Ad hoc Networking (MANET): Routing Protocol Performance Issues and Evaluation Considerations," RFC 2501 of IETF Network Working Group, Jan. 1999.

[5] P. Johansson, T. Larsson, N. Hedman, B. Mielczarek and M.Degermark, "Scenario-based Performance Analysis of Routing Protocols for Mobile Ad-hoc Networks, "International Conference on Mobile Computing and Networking (MobiCom'99), pp. 195-206, 1999.

[6] K. Arulanandam and Dr. B. Parthasarathy, "A New Energy level Efficiency Issues in MANET”, 2009.

[7] D. T. Ahmed and S. Shirmohammadi, Architectural Analysis of Multicast Routing Protocols for Wireless Ad Hoc Networks, Proceedings of the 6th International Conference on Networking, p. 21,2007.

[8] De Rango F., Guerriero F., Marano S., Bruno E. (2006). A Multiobjective Approach for Energy Consumption and Link Stability Issues in Ad Hoc Networks, IEEE. 